\title{
Urinary TWEAK Level as a Marker of Lupus Nephritis Activity in 46 Cases
}

\author{
Zhu Xuejing, ${ }^{1}$ Tan Jiazhen, ${ }^{1}$ Li Jun, ${ }^{1}$ Xu Xiangqing, ${ }^{1}$ Yuan Shuguang, ${ }^{1,2}$ and Liu Fuyou ${ }^{1}$ \\ ${ }^{1}$ Division of Nephrology, The Second Xiangya Hospital, Central South University, Changsha 410011, China \\ ${ }^{2}$ Kidney Institute of Central South University, The Second Xiangya Hospital, 139 Renmin Road, Hunan, Changsha 410011, China
}

Correspondence should be addressed to Yuan Shuguang, zhuxuejing5225209@163.com

Received 23 March 2012; Revised 13 April 2012; Accepted 13 April 2012

Academic Editor: Sergio O. De Paula

Copyright ( 2012 Zhu Xuejing et al. This is an open access article distributed under the Creative Commons Attribution License, which permits unrestricted use, distribution, and reproduction in any medium, provided the original work is properly cited.

\begin{abstract}
Objective. This study is designed to observe the urinary tumor necrosis factor-like weak inducer of apoptosis (TWEAK) levels in patients with lupus nephritis (LN) and to identify new biomarker of lupus nephritis activity. Methods. Study subjects were 46 cases of patients with LN (including 34 of active cases) who underwent routine renal biopsy. Activity and chronicity indexes of LN were assessed using pathological criteria proposed by Hill et al. in 2000. Urinary TWEAK (uTWEAK) level and Monocyte chemoattractant protein-1 (MCP-1) level were detected by ELISA. Results. Urinary TWEAK level was significantly higher in active LN group than in non-active LN group. Correlation analysis showed that urinary TWEAK levels were significantly correlated with activity index $(r=0.825, P<0.01)$, glomerular activity index $(r=0.754, P<0.01)$, and tubulointerstitial qualitative activity index $(r=0.751, P<0.01)$, while not significant correlated with chronicity Index $(P>0.05)$. The association between urinary TWEAK levels and urinary MCP-1 levels were significant in active LN group $(r=0.809, P<0.01)$ but not significant in non-active LN group $(P>0.05)$. Conclusions. uWEAK levels were correlated with all active indexes of LN, suggesting its potential role as novel biomarker of active lupus nephritis.
\end{abstract}

\section{Introduction}

Systemic lupus erythematosus (SLE) is a chronic autoimmune disease characterized by complex clinical manifestation and chronic inflammatory processes involving autoimmunity against multiple systems and multiple organs. Kidney is one of the most commonly affected organs. Lupus nephritis (LN), the appearance of a disservice caused by SLE to kidney, contributes to high mortality. As active stage and rest stage alternate iteratively in LN disease process, observation to SLE activity, especially to the level of renal activity, is extremely important to therapeutic regimen decisions and treatment modifications as well as prognosis. At present, activity of SLE is assessed based on clinical symptoms and biochemistry parameters such as autoantibody (e.g., anti-dsDNA) and serum complement. However, current biomarkers are not specific for evaluating renal activity. Renal biopsy has been the gold standard for assessment of kidney damage and disease activity, but its invasive feature has restricted its use.
Recently there has been a surge of interest in defining novel LN biomarkers to evaluate disease activity $[1,2]$. Over the last few years, evidences showed that tumor necrosis factor-like weak inducer of apoptosis (TWEAK) was expressed in a high level in the kidney of LN animal model. Patients of LN in active stage had higher urinary TWEAK level [3]. Researchers presumed that urinary TWEAK level may reflect LN activity. This study approached to the relationship between urinary TWEAK level and the activity of LN in the Asian patients in China.

\section{Patients and Methods}

2.1. Patients. Forty-six cases of patients with LN (12 of nonactive LN and 34 of active LN) were selected from patients hospitalized in the Second Xiangya Hospital from October 2008 to March 2009. Of those 34 cases have routine renal biopsy. The patients aged $14 \sim 53$ years with a mean duration of disease for $10.47 \pm 4.12$ months. Every case meets at least 4 items of SLE diagnostic criteria revised by American 
Rheumatism Association in 1982. All the patients received no treatment of immunosuppressive agent such as methylprednisolone (MP), cyclophosphamide (CTX), mycophenolate (MMF), ciclosporin A (CsA), or tacrolimus (FK506) for 3 months prior to the diagnoses of LN by renal biopsy. Occasional use of hydroprednisone in some patients was subjected to short time and low doses $(<30 \mathrm{mg} / \mathrm{d})$. Biopsy specimens were fixed, sectioning and staining (including HE, PAS, PASM, and Masson), then observed under light microscopy. $10 \mathrm{~mL}$ urina sanguinis from each patient was centrifuged. Then clear supernatant liquid was used for TWEAK and MCP-1 detection.

2.2. SLE Disease Activity Index Assessment. For renal involvement, renal SLE Disease Activity Index (SLEDAI) was used to assess kidney disease activity. The score consists of the four kidney-related parameters: hematuria, pyuria, proteinuria, and urinary casts. Scores for the renal SLEDAI can range from 0 (inactive renal disease) to a maximum of 16. A SLEDAI score of $\geqslant 4$ was taken as an indicator of active lupus nephritis.

According to the 2000 SLEDAI scores [4], patients were divided into two groups: nonactive group and active group. Nonactive group: 12 cases ( 3 males and 9 females), LN duration of $14.06 \pm 4.55$ months; active group: 34 cases ( 1 male and 33 females), LN duration of $8.44 \pm 2.13$ months.

Renal biopsy specimens from LN patients were classified according to the World Health Organization (WHO) criteria: minimal changes (class I), mesangial alterations (class II), focal proliferative (class III), diffuse proliferative (class IV), and membranous (class V) glomerulonephritis.

2.3. Lupus Nephritis Activity Index and Chronicity Index. We made semiquantitative score for each renal biopsy specimen according to 2000 Hill's Biopsy Index scoring criteria for lupus nephritis [5]. Activity Index (AI) and Chronicity Index (CI) were calculated. Activity Index contained Glomerular Activity Index (GAI) and Tubulointerstitial Activity Index (TIAI).

(1) Components of Glomerular Activity Index: (1) glomerular proliferation; (2) polymorphonuclear leukocytes; (3) karyorrhexis/Fibrinoid necrosis; (4) cellular crescents; (5) hyaline deposits; (6) glomerular monocytes.

(2) Components of Tubulointerstitial Activity Index: (1) tubular cell pyknosis; (2) tubular nuclear "activation"; (3) tubular cell necrosis; (4) tubular cell flattening; (5) macrophages in tubular lumens; (6) epithelial cells in tubular lumens; (7) interstitial inflammation.

(3) Activity Index: AI = GAI + TIAI.

(4) Components of Chronicity Index: (1) glomerulosclerosis; (2) glomerular scar; (3) fibrous crescentic; (4) tubular atrophy; (5) interstitial fibrosis.

2.4. The Detections of Urinary TWEAK and Urinary MCP1 Levels. Urinary TWEAK levels and urinary MCP-1 levels were measured by human TWEAK ELISA kits and human MCP-1 ELISA kits from Xin Bo Sheng Bio-Technology Co., Ltd. Shenzhen, China. We measured absorbance A values by Microplate reader at $450 \mathrm{~nm}$ wavelength and then calculated urinary TWEAK concentrations and urinary MCP-1 concentrations according to the standard curve. Urinary TWEAK levels and urinary MCP-1 levels were normalized to urinary creatinine concentrations measured in the same spot urine, expressed as $\mathrm{pg} / \mathrm{mg}$ creatinine.

2.5. Statistical Analysis. SPSS 13.0 statistical package was used for data analysis. Measurement data consistent with normal distribution was expressed as $\chi \pm S$. LSD-t test was used for comparing two groups. Association among categorical variables was measured by Pearson's Chi-squared test. Spearman's analysis was used for correlations between quantitative data and semiquantitative data or between two semiquantitative data. $P$-value $<0.05$ was considered statistically different and $P$-value $<0.01$ as statistically significant.

\section{Results}

3.1. Clinical and Laboratory Findings of Lupus Nephritis Patients. SLE patients $(n=46)$ were recruited into two groups: SLE patients with active lupus nephritis (active group) and those patients with inactive or nonrenal involvement (nonactive group). Statistically, significant differences were found between both groups in the following variables: red blood cell count in urine $(/ \mathrm{HP}), 24 \mathrm{~h}$ urinary proteins $(\mathrm{g} / 24 \mathrm{~h})$, serum BUN $(\mathrm{mmol} / \mathrm{L})$, serum creatinine level $(\mu \mathrm{mol} / \mathrm{L}), \mathrm{C} 3(\mathrm{mmol} / \mathrm{L})$, and $\mathrm{C} 4(\mathrm{mmol} / \mathrm{L})$. Detailed comparisons between both groups are presented in Table 1.

3.2. Features of the Histopathology of Active Lupus Nephritis. The pathological changes of active lupus nephritis contain glomerular mesangial cells proliferation, cellular crescents, thickening of capillary walls, karyorrhexis, hyaline thrombi, and interstitial inflammatory infiltration (Figure 1).

3.3. The Relationship between TWEAK and LN Activity. To determine whether TWEAK correlates with LN activity, we compared urinary TWEAK levels of LN active group with nonactive group. Urinary MCP-1 levels were used for positive control. As presented in Figure 2, urinary TWEAK levels of LN active group were significantly higher than those of nonactive group $(10.29 \pm 1.81 \mathrm{pg} / \mathrm{mgCr}$ versus $2.14 \pm$ $0.30 \mathrm{pg} / \mathrm{mgCr}, P<0.01$ ). Urinary MCP-1 levels (uMCP1) of LN active group were also significantly higher than those of nonactive group $(103.60 \pm 15.79 \mathrm{pg} / \mathrm{mgCr}$ versus $20.19 \pm 2.93 \mathrm{pg} / \mathrm{mgCr}, P<0.01)$.

3.4. The Relationship between UTWEAK and LN Renal Biopsy Index. The urinary TWEAK levels of patients with LN had significantly positive correlation $(r=0.825, P<0.01)$ with Active Index $(\mathrm{AI})(P<0.01)$, including Glomerular Activity Index $(\mathrm{GAI})\left(r_{\mathrm{GAI}}=0.745, r_{\mathrm{TIAI}}=0.751\right)$ and those with Tubulointerstitial Activity Index (TIAI), but had no 
TABLE 1: Comparison between active group and nonactive group in terms of important clinical and laboratory findings.

\begin{tabular}{|c|c|c|}
\hline & $\begin{array}{c}\text { Active Group }(n=34) \\
\text { Mean } \pm \text { SD }\end{array}$ & $\begin{array}{c}\text { Nonactive Group }(n=12) \\
\text { Mean } \pm \text { SD }\end{array}$ \\
\hline Male/female ratio & $1 / 33$ & $3 / 9$ \\
\hline Disease duration of SLE (month) & $12.71 \pm 3.39$ & $17.99 \pm 6.46$ \\
\hline Disease duration of LN (month) & $8.44 \pm 2.13$ & $14.06 \pm 4.55$ \\
\hline Red blood cell count in urine (/HP) & $6.36 \pm 0.67$ & $1.88 \pm 0.61$ \\
\hline 24-h urinary proteins $(\mathrm{g} / 24 \mathrm{hr})$ & $3396 \pm 1107$ & $1166 \pm 408$ \\
\hline Serum BUN (mmol/L) & $10.13 \pm 1.02$ & $7.84 \pm 2.11$ \\
\hline Serum $\operatorname{Cr}(\mu \mathrm{mol} / \mathrm{L})$ & $113.50 \pm 9.23$ & $92.36 \pm 19.09$ \\
\hline Serum C3 (mmol/L) & $0.46 \pm 0.04$ & $0.58 \pm 0.07$ \\
\hline Serum C4 (mmol/L) & $0.10 \pm 0.01$ & $0.14 \pm 0.02$ \\
\hline
\end{tabular}

SLE: systemic lupus erythematosus; LN: lupus nephritis; BUN: blood urea nitrogen; Cr: creatinine; C3: complement3; C4: complement4.

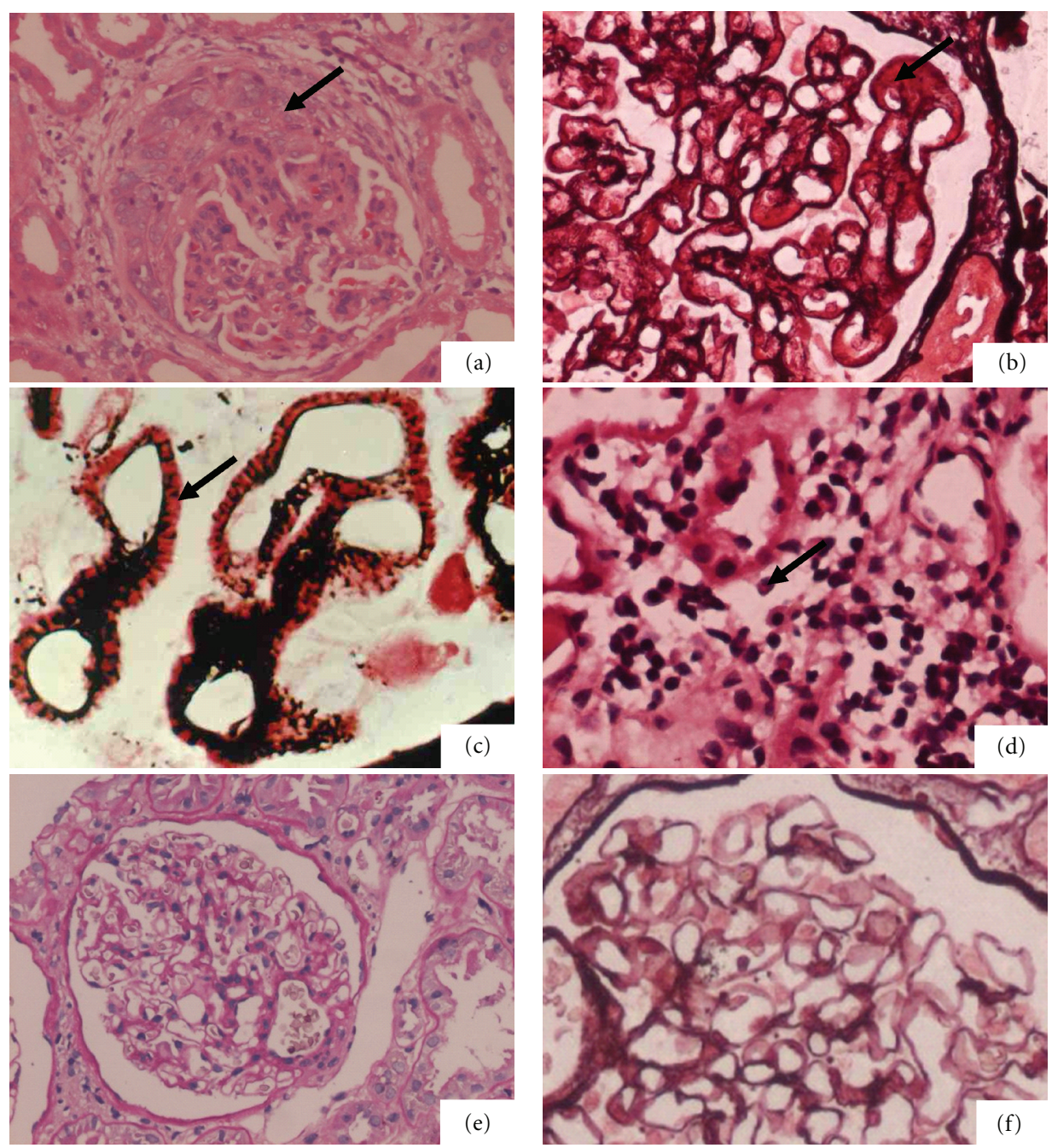

FIGURE 1: Features of the histopathology of active lupus nephritis. (a) Glomerular mesangial cells proliferation accompanied with cellular crescents (arrow showed). (HE staining, $\times 200$ ). (b) Subepithelial immune deposits to form a "platinum ear" (arrow showed). (PASM staining, $\times 200$ ). (c) Immune deposits in basement membrane. The "spikes bristle" can be observed in basement membrane (arrow showed). (PASM staining, $\times 1000)$. (d) Renal tubular epithelial cells shed accompanied with interstitial inflammatory infiltration (arrow showed). (HE staining, $\times 400$ ). (e) Normal glomerulus without mesangial cells proliferation. (PAS staining, $\times 200)$. (f) Normal glomerulus without thick basement membrane. (PASM staining, $\times 1000)$. 

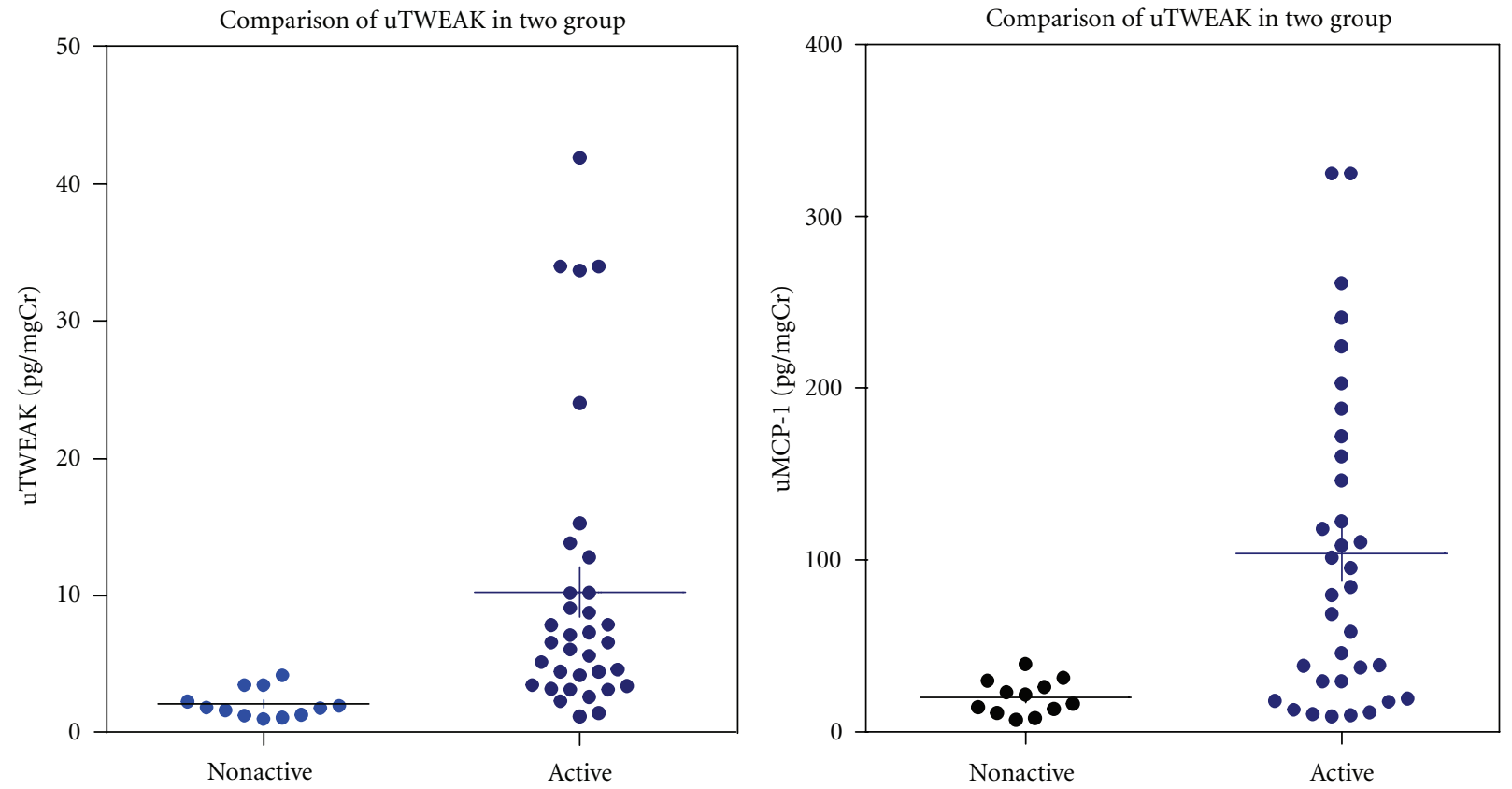

FIGURE 2: UTWEAK and uMCP-1 correlate with LN activity. A comparison of uTWEAK and uMCP-1 between 34 cases and 12 nonactive cases. Patients with active renal disease had significantly higher urinary TWEAK levels and higher urinary MCP-1 levels. Bars indicate median values.
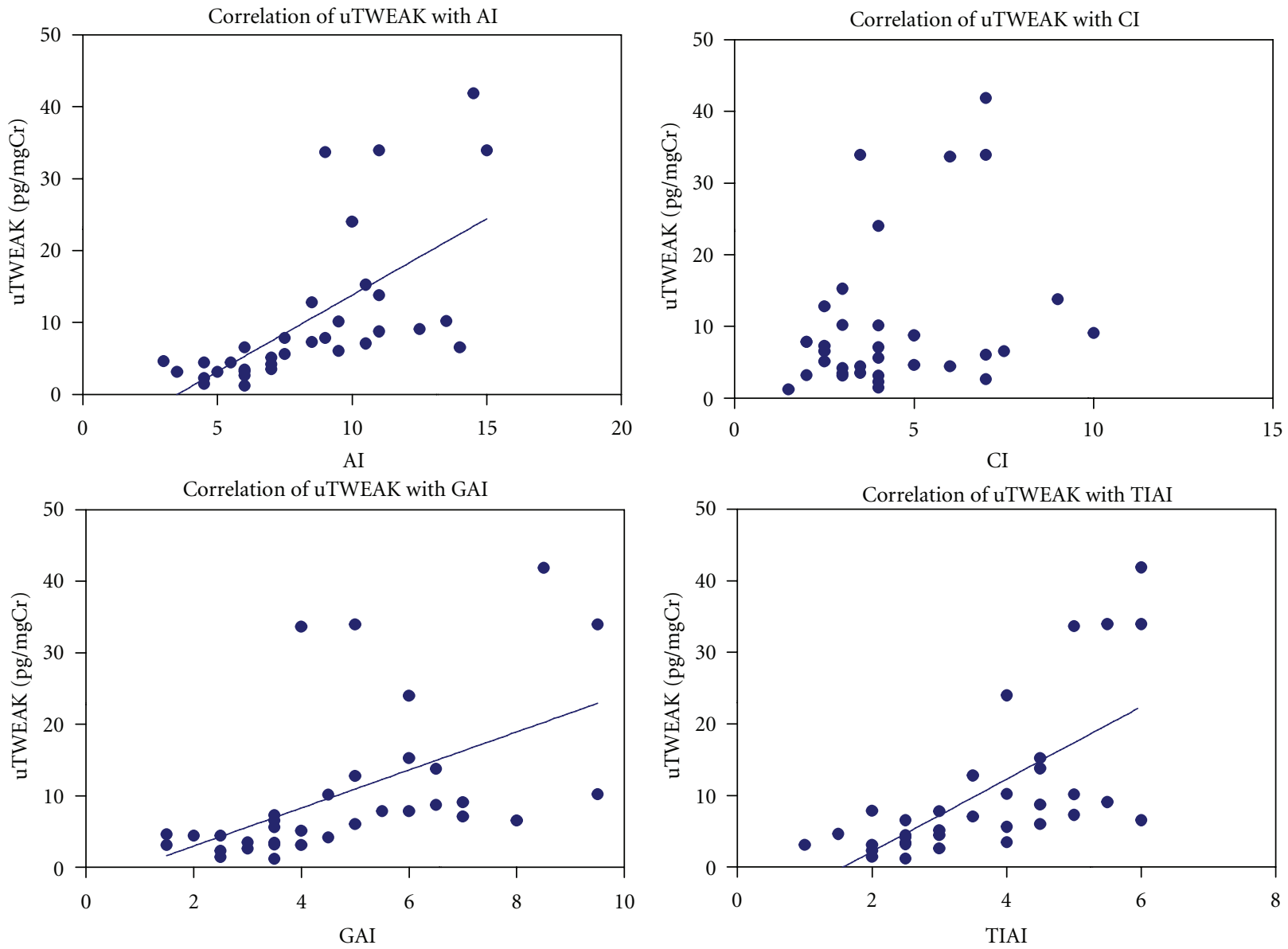

FIGURE 3: uTWEAK levels correlated with LN renal biopsy index. uTWEAK levels of 34 patients with LN who had routine renal biopsies showed positive correlations with Active Index (AI) $(r=0.825, P<0.01)$, Glomerular Activity Index $(\mathrm{GAI})(r=0.745, P<0.01)$, and Tubulointerstitial Activity Index (TIAI) $(r=0.751, P<0.01)$. uTWEAK levels had no correlation with Chronicity Index $(P>0.05)$. 

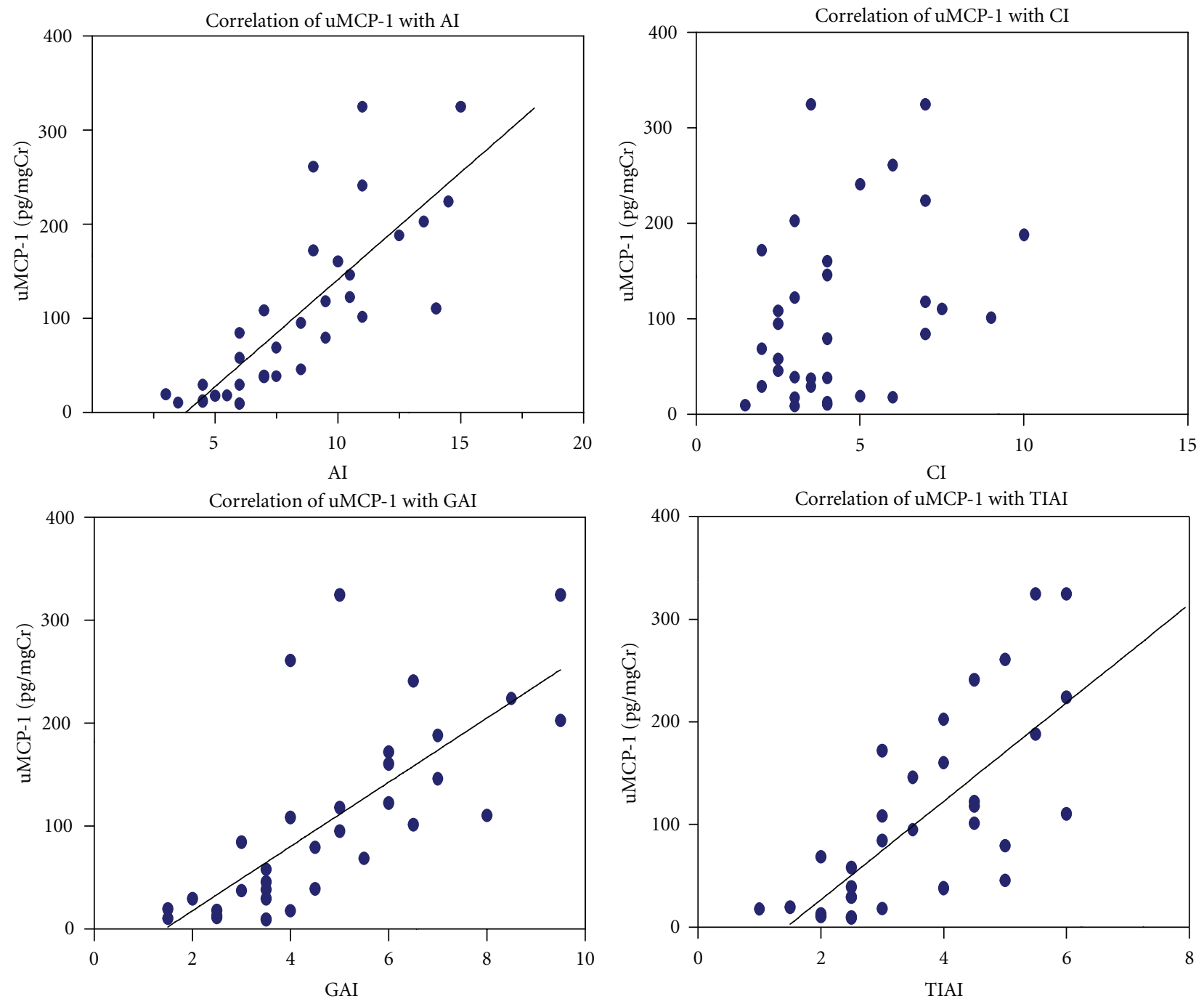

Figure 4: uMCP-1 levels correlated with LN renal biopsy index. A positive correlation $(r=0.800, P<0.01)$ between Active Index (AI) and uMCP-1 levels had been found from data of 34 patients with LN who had routine renal biopsies. And the analysis of urinary TWEAK levels with Glomerular Activity Index (GAI) and those with Tubulointerstitial Activity Index (TIAI) both yield positive $\left(r_{\mathrm{GAI}}=0.804\right.$, $\left.r_{\text {TIAI }}=0.769\right)$, significant $(P<0.01)$ correlations.

significant correlation $(P>0.05)$ with Chronicity Index $(\mathrm{CI})$ (Figure 3).

3.5. The Relationship between Monocyte Chemoattractant Protein-1 (MCP-1) and LN Activity. Monocyte chemoattractant protein-1 (MCP-1) has been presumed a biomarker to monitor LN activity, proved by evidences of biochemistry and immunology. However, MCP-1 was not reported to predict renal histopathology. This study analyzed the correlation between urinary MCP-1 levels and LN renal biopsy index. Results showed that the urinary MCP-1 levels of patients with LN had significantly positive correlation $(r=0.800, P<$ 0.01 ) with Active Index (AI), including Glomerular Activity Index (GAI), and those with Tubulointerstitial Activity Index (TIAI) $\left(r_{\mathrm{GAI}}=0.804, r_{\mathrm{TIAI}}=0.769\right)$ but had no significant correlation $(P>0.05)$ with Chronicity Index $(\mathrm{CI})$ (Figure 4).
3.6. The Relationship between Urinary TWEAK Levels and Urinary MCP-1 Levels. Relationship of urinary TWEAK levels with urinary MCP-1 levels was also analyzed. Our analysis showed that the urinary TWEAK levels in active group had significantly positive correlation $(r=0.809, P<$ 0.01 ) with urinary MCP-1 levels, while nonactive group's urinary TWEAK levels had no correlation with the urinary levels of MCP-1 $(P>0.05)$ (Figure 5).

\section{Discussion}

LN is one of the most common complications of SLE. In recent years, several studies have found that a disorder of the regulation of apoptosis is an important factor in SLE progress, such as TWEAK [6]. TWEAK combined with its receptor Fn14 could activate I $\kappa$-B kinase (IKK), resulting in the degradation and activation of $\mathrm{I} \kappa-\mathrm{B}$. I $\kappa-\mathrm{B}$ 

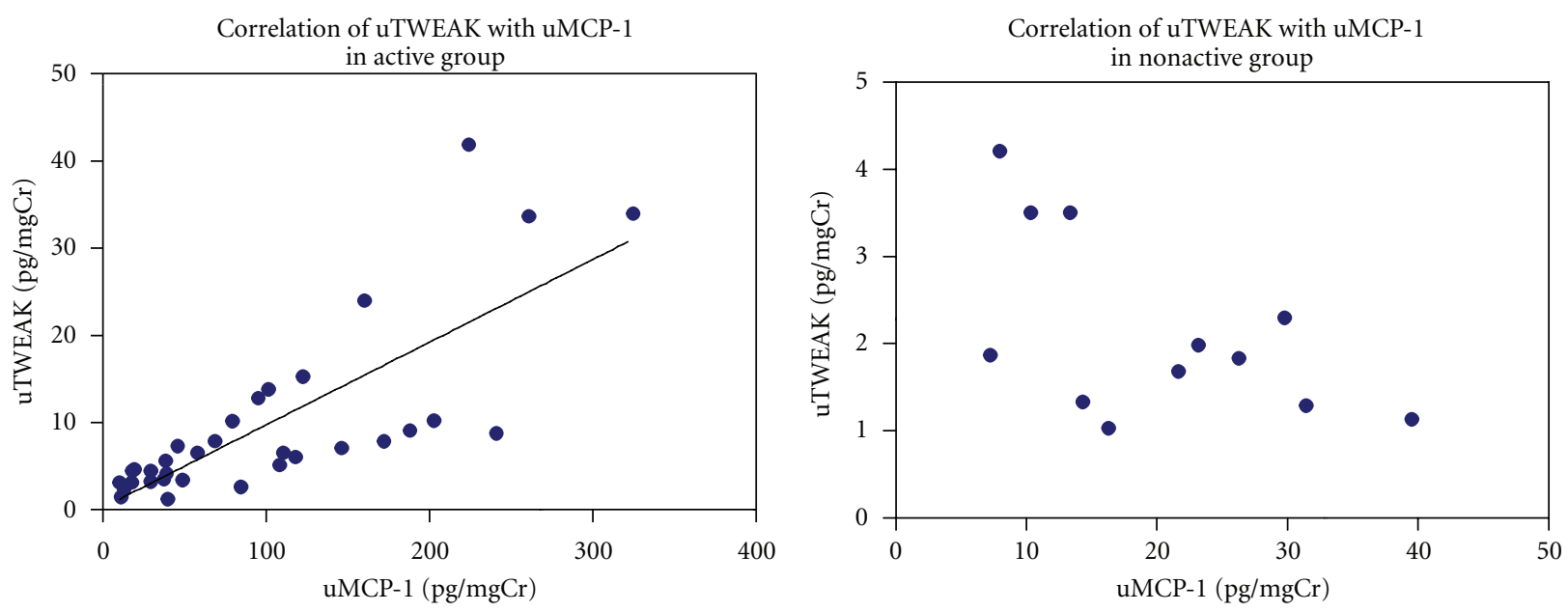

FIGURE 5: uTWEAK correlated with uMCP-1. Urinary TWEAK levels of 34 patient with active LN had significantly positive correlation with urinary MCP-1 levels, $r=0.809, P<0.01$. In disease nonactive group, 12 patients' urinary TWEAK levels had no correlation with their urinary MCP-1 levels.

transferred to cell nucleus and increased MCP-1, RANTES, and IL-6 expression, causing inflammatory cell infiltration. Researches also reported that TWEAK/Fn14 induced apoptosis of glomerular mesangial cells and tubular epithelial cells with induction of proinflammatory cytokines, thereby causing glomerular and tubular injury, which might play an important role in the pathogenesis of lupus nephritis $[7,8]$. In this study, we analyzed the relationship between TWEAK and the renal activity of lupus in order to prove TWEAK as a marker of lupus nephritis.

Previous studies have shown that serum TWEAK levels are significantly lower in SLE patients than in healthy individuals $[9,10]$. A cross-sectional study by Gladman et al. [4] in the Ohio SLE Research Center and the Albers Albert Einstein Medical College showed that patients with lupus nephritis had significantly higher urinary TWEAK levels than those without kidney injury, and the patients in active stage of lupus, with kidney injury or not, had higher urinary TWEAK levels than those in nonactive stage, indicating that an elevated level of urinary TWEAK may suggest active lupus nephritis. In our study, active LN patients had significantly higher urinary TWEAK levels, compared to those in chronic, stable condition. This result was similar to the findings of Schwartz et al. [11] focused on the cases in USA. And our study provides a supporting result to the conclusion by Schwartz et al. in Chinese patients, indicating that TWEAK also played an important role in the active lupus nephritis in Asian cases.

In this study, the correlation between MCP-1 and LN activity has been confirmed by histopathological evidence. Results also showed that urinary TWEAK levels had significantly correlated with LN renal pathology AI containing GAI and TIAI. However, no relationship was found between urinary TWEAK levels and CI. This indicates that urinary TWEAK levels could reflect the level of activity in LN patients. In addition, the expression of TWEAK is relevant to glomerular lesions and tubulointerstitial lesions. The elevation of urinary TWEAK level may be related to the increased expression of TWEAK in kidney. However, it is still uncertain whether kidney is the only organ expressing TWEAK, which is needed to be confirmed in the future. We also found that urinary TWEAK levels have no difference between Class II, III, and IV of LN among 31 patients (Result is not showed in this article), indicating that urinary TWEAK detection cannot replace renal biopsy entirely in diagnostic process.

MCP-1 was proved to be a biomarker of lupus nephritis disease activity $[12,13]$. In vivo, urinary MCP-1 has positive correlation with the increase in urine $n$-acetyl- $\beta$-dglucosaminidase (NAG) and urine protein in $24 \mathrm{~h}$ [14]. A significantly positive correlation between MCP-1 and AI has been showed in our study (Figure 4). In addition, urinary TWEAK levels have significantly positive correlation with active LN $(r=0.809, P<0.01)$ and urinary levels of MCP1 , pointing to the potential of urinary TWEAK as a diseasemonitoring biomarker. Activated $\mathrm{T}$ lymphocytes expressed TWEAK, resulting in increasing apoptosis of mononuclear cells. The injured mononuclear cells released nuclear selfantigens by increasing MCP-1 expression, and the clearance of apoptotic debris reduced relatively, which might be one of the mechanisms for the development of lupus. Researches indicated that TWEAK combined with Fn14 induced MCP-1 in renal tubular epithelial cells, causing RelA subunit of NF$\kappa \mathrm{B}$ transferring from cytoplasm to nucleus, leading to the activation of target gene which encodes MCP-1 $[6,15,16]$. These results proved that TWEAK is one of the upstream regulatory factors of MCP-1 in inflammatory process.

In conclusion, urinary TWEAK levels of patients with active LN significantly positively correlated with urinary levels of MCP-1, indicating the potential disease-monitoring biomarker of urinary TWEAK.

\section{Author Contribution}

Z. Xuejing and T. Jiazhen equally contributed to the work included in this paper. 


\section{Acknowledgment}

This paper was supported by the Fundamental Research Funds for the Central Universities (2012QNZT146).

\section{References}

[1] B. H. Rovin, H. Song, D. J. Birmingham, L. A. Hebert, C. Y. $\mathrm{Yu}$, and H. N. Nagaraja, "Urine chemokines as biomarkers of human systemic lupus erythematosus activity," Journal of the American Society of Nephrology, vol. 16, no. 2, pp. 467-473, 2005.

[2] Y. Li, M. Tucci, S. Narain et al., "Urinary biomarkers in lupus nephritis," Autoimmunity Reviews, vol. 5, no. 6, pp. 383-388, 2006.

[3] N. Schwartz, C. Putterman, L. H. Su et al., "Urinary TWEAK and the activity of lupus nephritis," Journal of Autoimmunity, vol. 27, no. 4, pp. 242-250, 2006.

[4] D. D. Gladman, D. Ibañez, and M. B. Urowltz, "Systemic lupus erythematosus disease activity index 2000," The Journal of Rheumatology, vol. 29, no. 2, pp. 288-291, 2002.

[5] G. S. Hill, P. Chedin, D. Nochy et al., "A new morphologic index for the evaluation of renal biopsies in lupus nephritis," Kidney International, vol. 58, no. 3, pp. 1160-1173, 2000.

[6] J. A. Winkles, "The TWEAK-Fn14 cytokine-receptor axis: discovery, biology and therapeutic targeting," Nature Reviews Drug Discovery, vol. 7, no. 5, pp. 411-425, 2008.

[7] A. B. Sanz, A. Ortiz, P. Justo et al., "The cytokine TWEAK modulates renal tubulointerstitial inflammation," Journal of the American Society of Nephrology, vol. 19, no. 4, pp. 695-703, 2008.

[8] H. X. Gao, S. R. Campbell, L. C. Burkly et al., "TNF-like weak inducer of apoptosis (TWEAK) induces inflammatory and proliferative effects in human kidney cells," Cytokine, vol. 46, no. 1, pp. 24-35, 2009.

[9] S. Kralisch, M. Ziegelmeier, A. Bachmann et al., "Serum levels of the atherosclerosis biomarker sTWEAK are decreased in type 2 diabetes and end-stage renal disease," Atherosclerosis, vol. 199, no. 2, pp. 440-444, 2008.

[10] J. J. Carrero, A. Ortiz, A. R. Qureshi et al., "Additive effects of soluble TWEAK and inflammation on mortality in hemodialysis patients," Clinical Journal of the American Society of Nephrology, vol. 4, no. 1, pp. 110-118, 2009.

[11] N. Schwartz, T. Rubinstein, L. C. Burkly et al., "Urinary TWEAK as a biomarker of lupus nephritis: a multicenter cohort study," Arthritis Research \& Therapy, vol. 11, no. 5, article R143, 2009.

[12] C. Zoja, X. Liu, R. Donadelli et al., "Renal expression of monocyte chemoattractant protein-1 in lupus autoimmune mice," Journal of the American Society of Nephrology, vol. 8, no. 5, pp. 720-729, 1997.

[13] T. Wada, H. Yokoyama, S. B. Su et al., "Monitoring urinary levels of monocyte chemotactic and activating factor reflects disease activity of lupus nephritis," Kidney International, vol. 49, no. 3, pp. 761-767, 1996.

[14] J. H. Yang, Q. L. Zhou, W. N. Nie et al., "Clinical significance of monitoring of monocyte chemoattractant protein-1 in lupus nephritis," Journal of Clinical Research, vol. 12, pp. 2069-2070, 2007.

[15] P. Saas, J. Boucraut, P. R. Walker et al., "TWEAK stimulation of astrocytes and the proinflammatory consequences," Glia, vol. 32 , no. 1, pp. 102-107, 2000.
[16] P. Justo, A. B. Sanz, M. D. Sanchez-Niño et al., "Cytokine cooperation in renal tubular cell injury: the role of TWEAK," Kidney International, vol. 70, no. 10, pp. 1750-1758, 2006. 


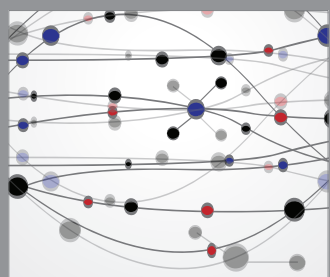

The Scientific World Journal
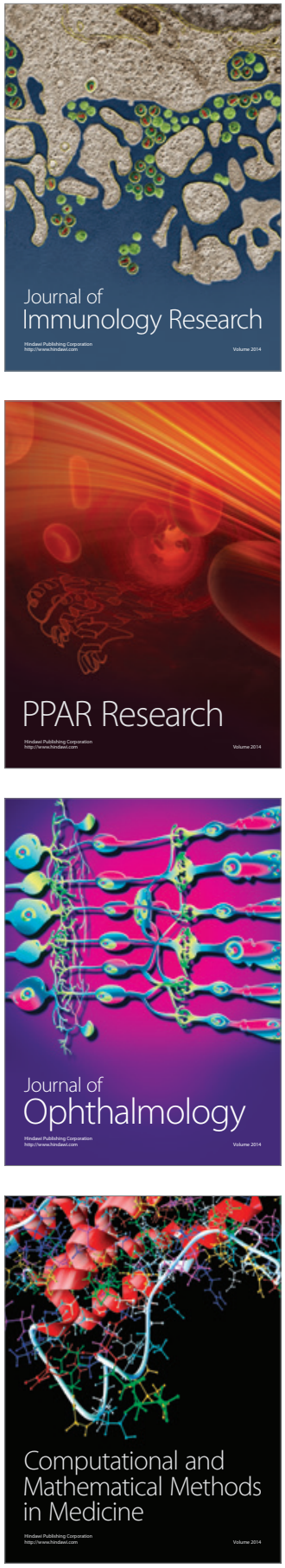

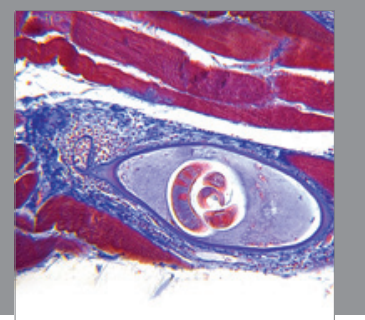

Gastroenterology

Research and Practice
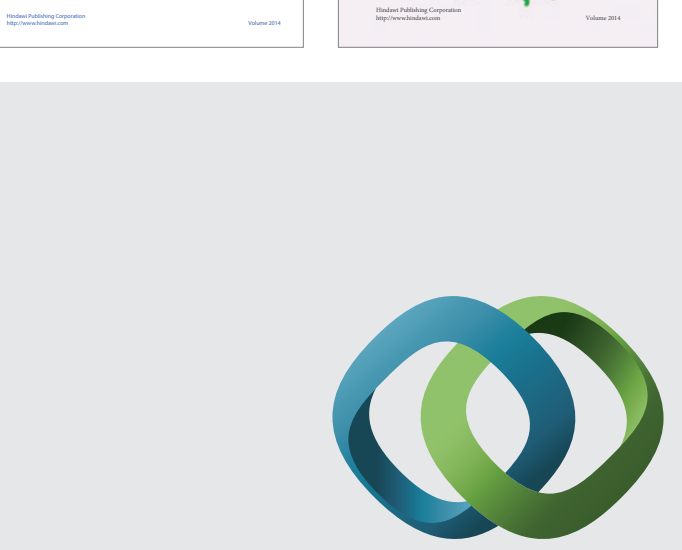

\section{Hindawi}

Submit your manuscripts at

http://www.hindawi.com
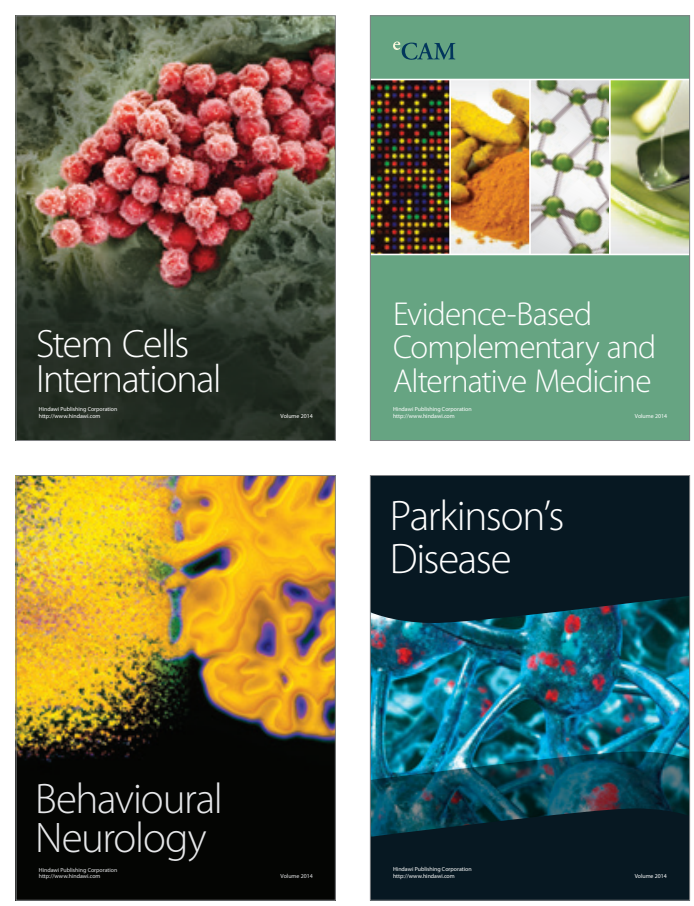

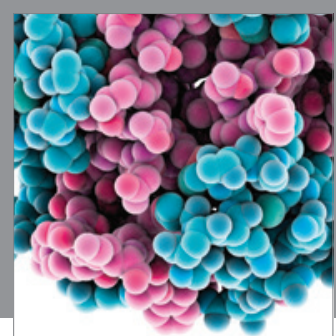

Journal of
Diabetes Research

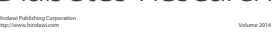

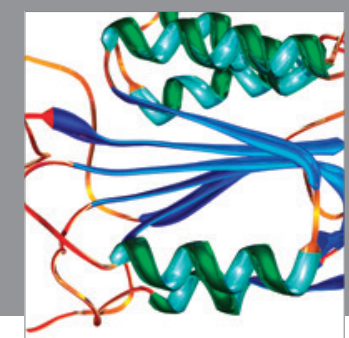

Disease Markers
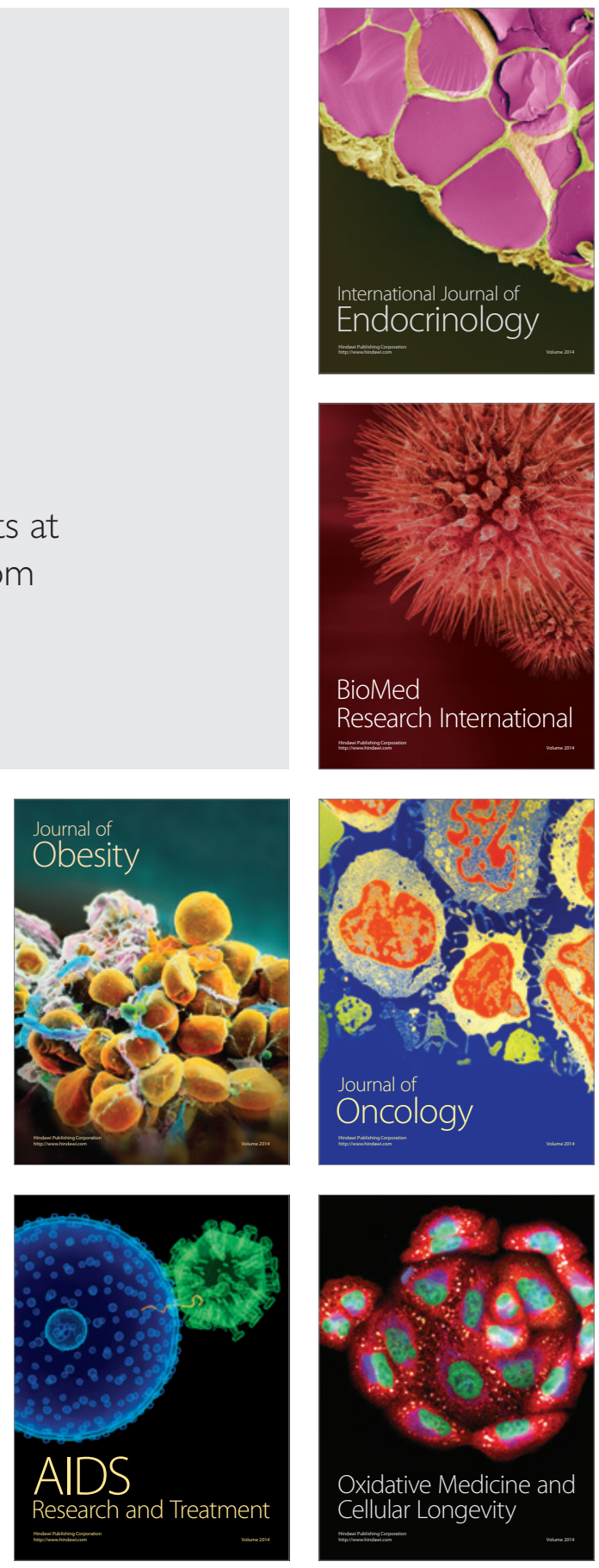